\title{
Processing of Ultrafine-Size Particulate Metal Matrix Composites by Advanced Shear Technology
}

\begin{abstract}
N.S. BAREKAR, S. TZAMTZIS, N. HARI BABU, Z. FAN, and B.K. DHINDAW
Lack of efficient mixing technology to achieve a uniform distribution of fine-size reinforcement within the matrix and the high cost of producing components have hindered the widespread adaptation of particulate metal matrix composites (PMMCs) for engineering applications. A new rheo-processing method, the melt-conditioning high-pressure die-cast (MC-HPDC) process, has been developed for manufacturing near-net-shape components of high integrity. The MC-HPDC process adapts the well-established high shear dispersive mixing action of a twin-screw mechanism to the task of overcoming the cohesive force of the agglomerates under a high shear rate and high intensity of turbulence. This is followed by direct shaping of the slurry into near-net-shape components using an existing cold-chamber die-casting process. The results indicate that the MC-HPDC samples have a uniform distribution of ultrafine-sized SiC particles throughout the entire sample in the as-cast condition. Compared to those produced by conventional high-pressure die casting (HPDC), MC-HPDC samples have a much improved tensile strength and ductility.
\end{abstract}

DOI: $10.1007 / \mathrm{s} 11661-008-9750-8$

(C) The Minerals, Metals \& Materials Society and ASM International 2009

\section{INTRODUCTION}

DUE to attractive physical and mechanical properties, metal matrix composite (MMC) materials have emerged as potential contenders in the arena of engineering materials. The unified combination of metallic properties of the matrix with the ceramic properties of the reinforcement, such as high specific modulus, high specific strength, and thermal stability, MMCs have the potential to serve the spectrum of applications in aerospace, automotive, electronic, and recreation industries. $^{[-7]}$ The focus has increasingly shifted toward discontinuously-reinforced composites as a competition to continuous fiber reinforced composites from the standpoint of isotropic mechanical properties. ${ }^{[1-7]}$

The concept and advantages of reinforcing a metal are now very well established. Particulate metal matrix composites (PMMCs) have been shown to offer improvements in strength, wear resistance, structural efficiency, reliability, and control of physical properties such as density and coefficient of thermal expansion. ${ }^{[6]}$ To obtain a specific mechanical/physical property, ideally speaking, the MMCs should consist of ultrafine (particle size $<10 \mu \mathrm{m}$ ) particulates distributed uniformly

N.S. BAREKAR, Visiting Doctoral Student, BCAST (Brunel Centre for Advanced Solidification Technology), Brunel University, is Research Scholar, Department of Metallurgical and Materials Engineering, Indian Institute of Technology. B.K. DHINDAW, Professor, is with the Department of Metallurgical and Materials Engineering, Indian Institute of Technology, Kharagpur-721302, India. Contact e-mail: dhindaw@metal.iitkgp.ernet.in S. TZAMTZIS, Doctoral Student, N. HARI BABU, Lecturer, and Z. FAN, Professor, are with BCAST (Brunel Centre for Advanced Solidification Technology), Brunel University, Uxbridge, Middlesex UB8 3PH, United Kingdom.

Manuscript submitted March 14, 2008.

Article published online January 17, 2009 in a ductile matrix with clean interface between the particulate and matrix. ${ }^{[6]}$ The strength of PMMCs increases with decreasing reinforcement size, but on the other hand, fine size and large volume fractions lead to severe agglomeration of the discontinuous reinforcement. A fine particle size has been attributed to an increase in the total surface area of the particulates, which increases the tendency for them to clump together ${ }^{[8]}$ The clustering of reinforcement particles in cast composites has a number of deleterious effects on their mechanical properties. Clusters act as crack or decohesion nucleation sites, or both, at stresses lower than matrix yield strength, causing the MMC to fail at unpredictable low stress levels. ${ }^{[9,10]}$

The MMCs are generally processed with liquid metal routes such as stir casting and infiltration. A powder metallurgy route is also used for specific applications. ${ }^{[28]}$ However, the infiltration route is the most commonly used method by industry and accounts for the largest volume in primary production. ${ }^{[1,3]}$ This method is not suitable to produce fine-sized PMMCs with a wide variety of complex shapes as it requires extremely high pressure to infiltrate the liquid metal into fine-sized particle preforms. Increased processing steps add to the cost and aid chemical reactions between the matrix and particle, which often result in brittle secondary phases. ${ }^{[1]}$

Because of the ease of operation and attractive economical aspects, stir casting is extensively used to synthesize composites reinforced with particulates of a wide variety of ceramic materials. The major problem in the synthesis of PMMCs by stir casting is to obtain a homogeneous distribution of the reinforcing phase. In composites produced by this method, the particle distribution will change significantly depending on process parameters during both the melt and solidification stages 
of the process. Several authors have tried to optimize the process parameters in order to obtain a uniform distribution of particulates throughout the melt. ${ }^{[12-15]}$ Much research has been reported about the particulate distribution in the matrix of PMMCs, as affected by the interaction between the solidification conditions and particulates. ${ }^{[16-20]}$ Even at relatively rapid solidification rates in pressure die casting a uniform particle distribution was not observed. ${ }^{[21-24]}$ This characterization of reinforcement distribution in cast composites has been primarily done on a subjective basis. Although the foregoing processes seem to generally identify particle clustering, the results are not strictly quantitative and there is an inherent lack of sensitivity to small variations of inhomogeneity caused by process variables. In the present study, effort has been undertaken to identify the influence of different processing variables on the resultant reinforcement distribution in cast composites. Apart from the degree of particulate homogeneity and cost effectiveness, the degree of wettability of reinforcement particles by the matrix alloy is another important aspect of liquid phase processing. ${ }^{\text {[25] }}$

For a given set of constituents, in PMMCs the fundamental link between microstructure and properties is determined by the fabrication method. It is now clear to the materials research community that if PMMCs are to gain industrial viability, their processing must be rendered economical and reliable and must be tailored to produce microstructures that will optimize critical properties of composites. ${ }^{[2-34]}$ However, with conventional processing methods, the particles accumulate in stagnant zones relatively undisturbed by fluid shear in the bulk and often produce agglomerates in the ductile matrix and, as a result, the composites exhibit extremely low ductility. ${ }^{[35,36]}$

Despite the continued work carried out, further efforts are required to improve the microstructure and enhance mechanical properties such as tensile strength, ductility, fracture toughness, etc. especially for reinforcement with ultrafine-size particulates. The severe agglomerative nature of ultrafine-sized particles due to their high cohesive energy, ${ }^{[37]}$ combined with a lack of dispersive technology for mixing poorly wettable particles, have hindered the progress in fabrication of high performance PMMCs with economically viable liquid processing routes.

These problems can be addressed by developing an efficient process for reducing the agglomeration and processing time in the liquid state. The key idea in this article is to overcome the average cohesive force or the tensile strength of the cluster embedded in the liquid metal by applying a sufficient shear stress $(\tau)$ during mixing. Even in the case of atoms connected with high bonding energy, molecular dynamic studies ${ }^{[38,39]}$ suggest that the intensive shearing can displace the position of atoms that are held together. In this article, we present a new rheo-processing technique, the melt conditioning by advanced shear technology (MCAST) ${ }^{[43]}$ capable of producing components of high integrity and improved performance, while being comparable with the highpressure die-casting (HPDC) process in terms of the production cost and efficiency. To date, due to the agglomerative nature of ultrafine-sized particles, largesized particulates have been extensively used as reinforcement. ${ }^{[21-24,32,33]}$ Current research concentrates on optimization, such as improving the uniformity of the reinforcement distribution of $\mathrm{SiC}$ (average size $4 \mu \mathrm{m}$ ) in an aluminum alloy (LM24/A380) under intensive shearing. In the liquid processing route, for the first time, an attempt has been made to fabricate PMMCs with such a fine $\mathrm{SiC}$ reinforcement. To assess the effect of processing variables on the $\mathrm{SiC}_{p}$ distribution and the effectiveness of the MCAST processing technique, results have been quantitatively investigated and compared. The experimental results on properties from tensile tests for the melt-conditioned high-pressure die-cast (MC-HPDC) Al alloy composites are also presented.

\section{MELT CONDITIONING: A RHEO PROCESS}

The process for producing low cost Al-MMC for casting primarily consists of two steps: (A) distributive mixing and (B) dispersive mixing under intensive shearing prior to shaping.

\section{A. Distributive Mixing}

The distribution of reinforcing particles is strongly influenced by many aspects of the processing route. ${ }^{[12-15]}$ Distributive mixing (Figure 1) employs stirring to premix the Al with SiC particles. According to Bernoulli's theorem, the negative pressure differential existing at the vortex helps to suck externally added particles into the liquid metal. The force provided by stirring the melt with a mechanical stirrer helps to overcome the surface energy barriers due to the poor wettability of $\mathrm{SiC}$ by $\mathrm{Al}$ alloy. Once the particles are transferred into the liquid, the distribution is strongly affected by certain flow transitions. The application of a shear force to the added

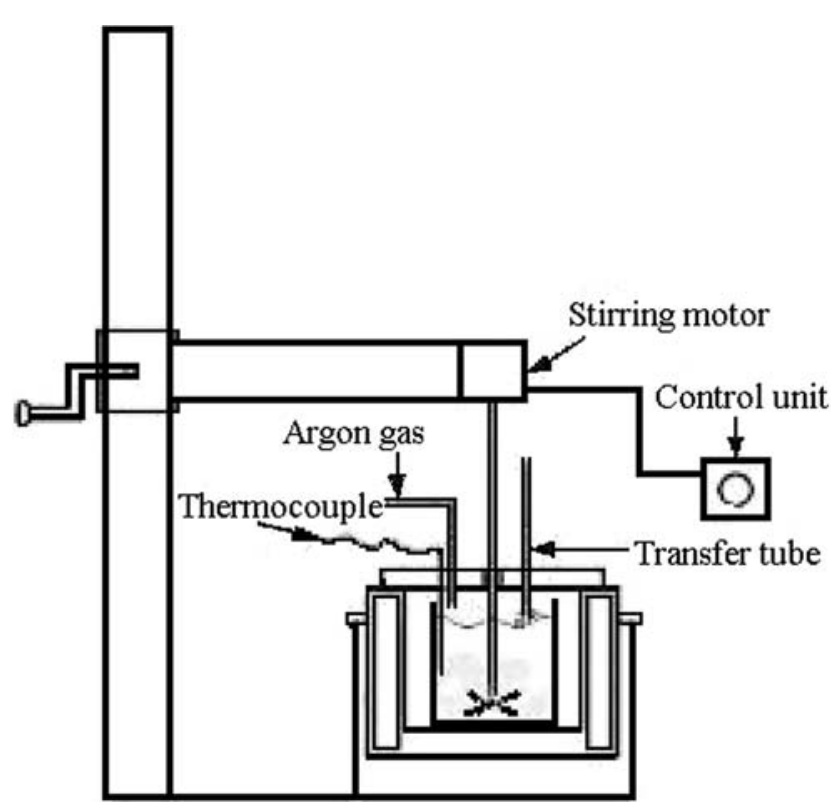

Fig. 1-Schematic diagram of distributive mixing equipment. 
particulates contributes to distribute them relatively uniformly. In order to produce adequate melt circulation and homogeneous distribution of reinforcement throughout the melt, the impeller design plays a crucial role. ${ }^{[40,41]}$ To avoid stagnant zones, necessary axial and radial flows are provided by an impeller with an impeller diameter/crucible diameter ratio of 0.4 , and a width of impeller/diameter of impeller ratio of $0.35{ }^{[14]}$ Stirring of the mixture is carried out in the liquid and semisolid regime to achieve spatial homogeneity of particulates (similar volume fraction in any part of the mixture).

The mixture is allowed to solidify in the crucible, remelted, and restirred before transferring to the next step of the mixing process. The force on a cluster is directly related to the viscosity of the medium. The maximum force on a particle cluster rotating in a shear flow $^{[40]}$ in the vicinity of a stirrer is given by

$$
F=6 \pi \eta a^{2} \dot{\gamma}
$$

where $a$ is the radius of each primary particle in the cluster, $\eta$ is the viscosity, and $\dot{\gamma}$ is the shear rate in the surrounding liquid medium. The degree of mixing is governed by the momentum transfer from the position of the stirrer to the clusters located away from the stirrer position. In this mixing step, due to the presence of velocity gradients within the liquid media, the shear force applied to the liquid that is in contact with the impeller results in a low shear force when averaged out over the entire volume of the liquid media. Thus, the degree of mixing is limited for the clusters located away from the impeller. In order to break up the agglomerates of ultrafine-sized $\mathrm{SiC}$ particles, it is important to increase the shear force.

\section{B. Dispersive Mixing Under Intensive Shearing}

To break up the agglomerates into individual particles in the liquid metal, the applied shear stress $(\tau)$ on particulate clusters should overcome the average cohesive force or the tensile strength of the cluster. Considering the agglomerate as a collection of spherical particles bonded to each other via an interparticle force $F$, Rumpf ${ }^{[8]}$ has calculated the tensile strength of an agglomerate $T$, and suggests that $\mathrm{T} \propto F_{c} / d^{2}$, where $d$ is the diameter of the individual particle. According to Kendall's model, ${ }^{[42]}$ the strength of agglomerate is given by

$$
T \propto \frac{\phi^{4} \Gamma_{c}^{5 / 6} \Gamma^{1 / 6}}{\sqrt{l_{f} D_{a}}}
$$

where $\phi, D_{a}, \Gamma_{c}$, and $\Gamma$ are the volume fraction of particulates, agglomerate size, fracture surface energy, and equilibrium surface energy, respectively, and $l_{f}$ is the flaw size in the cluster. According to this, for a smaller particle size and shorter interparticle distance, which is the case for a fine powder, the tensile strength of agglomerate is much higher. While cohesive forces hold the agglomerates together in ultrafine powder, high hydrodynamic forces are needed to break them up.

The process of mixing under intensive shearing adapts the well-established high shear dispersive action of a twin screw mechanism ${ }^{[43,44]}$ to the task of overcoming the cohesive force or the tensile strength of the cluster given by Eq. [2]. The twin-screw mechanism used in MCAST machine (Figure 2) consists of a pair of screws rotating inside a barrel. The screws have specially designed profiles, which are co-rotating, fully intermeshing, and self-wiping. The fluid flow during mixing is characterized by a high shear rate and high intensity of turbulence. The basic function of the twin screw is to break up the clusters embedded in the liquid melt under a high shear rate and disperse the particles uniformly under the high intensity of turbulence. This mixture is then transferred to the shot chamber of HPDC machine for component shaping (Figure 2). This melt-conditioning process has a shorter cycle time and is more efficient

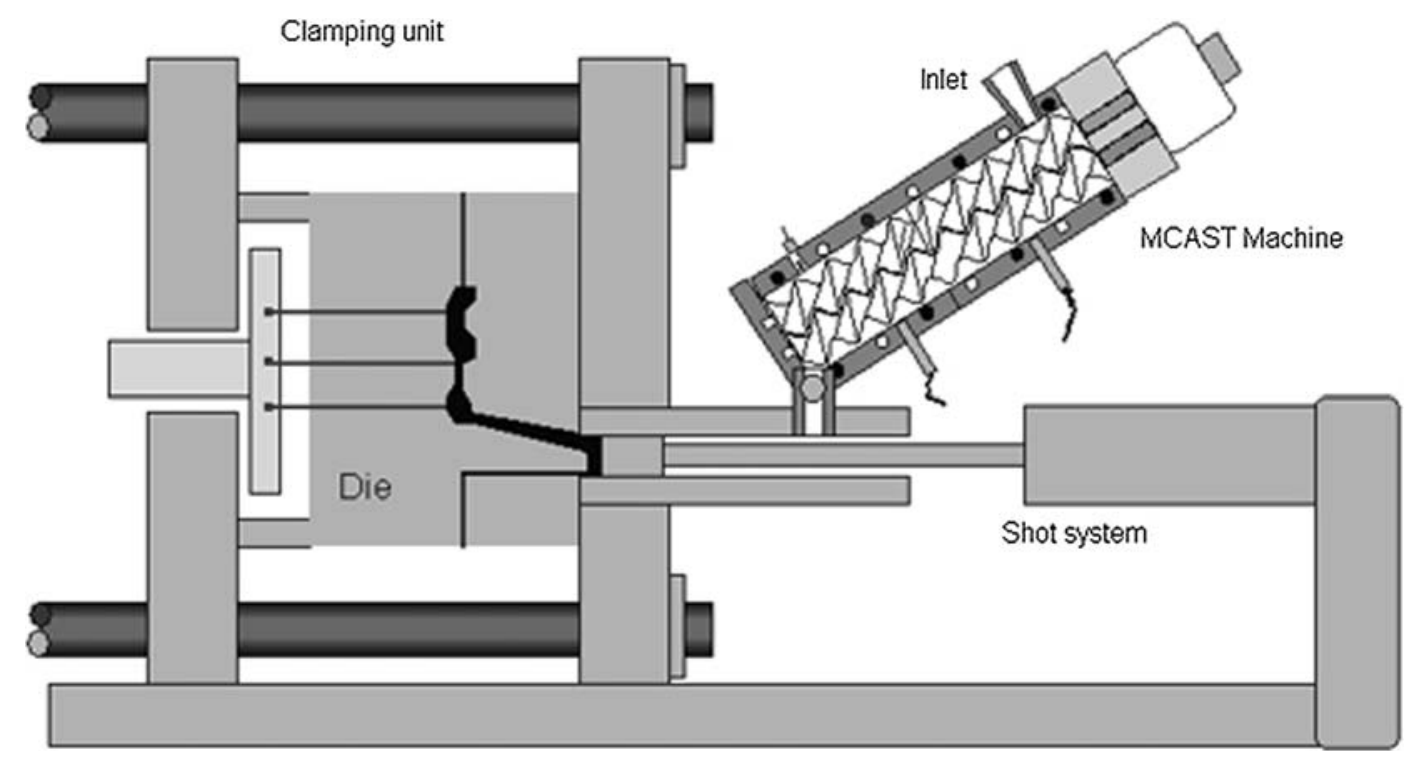

Fig. 2-Schematic illustration of the MC-HPDC process. 
than the conventional melt-stirring process. The melt conditioning in conjunction with high-pressure die casting results in a high efficiency and low production cost.

\section{EXPERIMENTAL}

\section{A. Distributive Mixing}

The matrix material used for the current study was LM24/A380, having the composition average values provided by the supplier (Norton Aluminium Ltd., Cannock, Staffordshire, United Kingdom) in weight percent, as shown in Table I.

Alloy LM24 (A380) has an excellent combination of mechanical properties in the cast condition and essentially the most common HPDC alloy. Interfacial reactions with reinforcement under a prolong contact time at elevated temperature is avoided due to the high $\mathrm{Si}$ content. ${ }^{[45]}$ The solidification synthesis of PMMCs involved introduction of a reinforcement into the melt and obtaining a suitable distribution by conventional mechanical stirring and dispersion by high-shear twinscrew mixing.

A properly cleaned ingot was melted in a cylindrical crucible (height of $200 \mathrm{~mm}$ and inner diameter of $90 \mathrm{~mm}$ ) inside a top-loaded resistance furnace at $650{ }^{\circ} \mathrm{C}$. With the help of a transfer tube, preheated (at $400{ }^{\circ} \mathrm{C}$ ) $\mathrm{SiC}$ ultrafine powder was added at the side of the vortex created by stirring the melt with the impeller rotating in clockwise direction. ${ }^{[14]}$ Then 5 and $10 \mathrm{vol}$ pet of SiC particles (average size $4 \mu \mathrm{m}$, supplied by Electro Abrasives, Buffalo, NY) were mixed with the LM24 (A380) alloy. The observed particle size distribution in the cast sample (Figure 3) was examined using optical microscope (Carl Zeiss Axioskop 2 MAT0) equipped with image analysis software. A four-bladed stainless steel impeller was coated with boron nitride to prevent the reaction with molten aluminum. A continuous purge of argon gas was maintained to minimize the oxidation of molten aluminum. Due to the poor wetting nature of $\mathrm{SiC}$ with liquid $\mathrm{Al}$, in order to enhance their incorporation stirring was carried out between the liquid and mushy state (liquidus temperature of LM24 $=593.4{ }^{\circ} \mathrm{C}$ ).$^{[46]} \mathrm{A}$ lifting mechanism for the rotational drive unit and stirrer assembly was used to extract the stirrer from the melt and to facilitate the stirrer positioning, cleaning, and replacement. The composite mixture thus obtained was allowed to solidify in the crucible and was subsequently remelted, restirred, and fed to a HPDC machine (DCC280, LK Machinery, Hong Kong), with a 280 tonnes clamping force to produce standard tensile test samples. Samples produced in this conventional process are referred as "HPDC" samples.

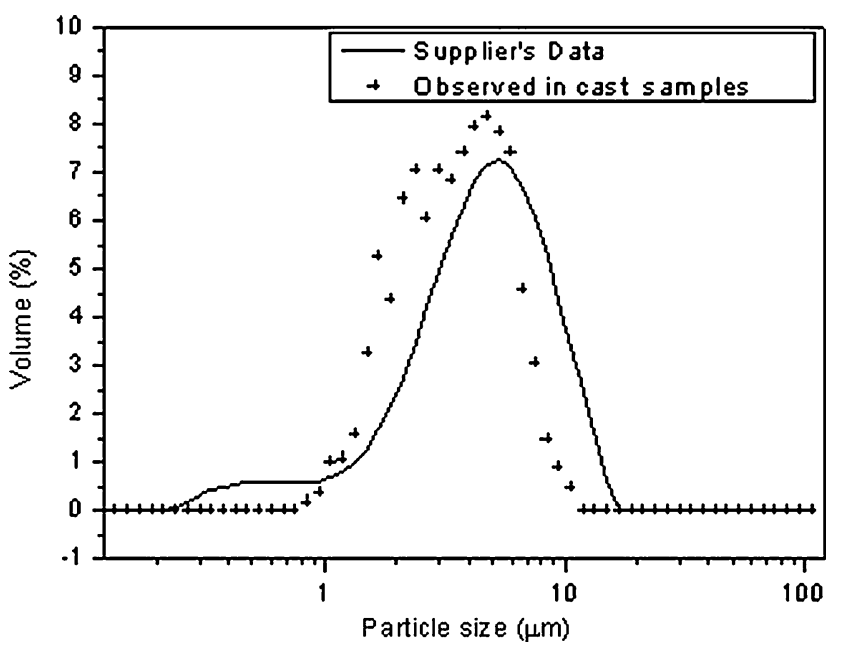

Fig. 3-SiC particle size distribution.

\section{B. Dispersive Mixing}

Melt-conditioning high-pressure die casting MCHPDC is a processing technique for manufacturing near-net-shape components of high integrity. The MCHPDC equipment consists of two basic functional units: a MCAST machine and a standard cold chamber HPDC machine. The premixed composite mixture after distributive mixing, as described, was fed into the slurry maker. A series of trial experiments were carried out to decide the range of process parameters. Process parameters varied during the experiments and reasons for selection are listed in Table II. The maximum temperature and speed limits in the MCAST machine are $650^{\circ} \mathrm{C}$ and $1000 \mathrm{rpm}$. The composite mixture was mechanically sheared by a pair of closely intermeshing screws for selected processing parameters and reinforcement particles were dispersed homogeneously throughout the melt. A more detailed description of fluid flow in the MCAST machine can be found in References 44 and 47. After shearing for a given period of time, the composite slurry was transferred to the HPDC machine with a 280 tonnes clamping force, to produce standard tensile test samples (MC-HPDC samples). The dimensions of the tensile test samples are $6.4 \mathrm{~mm}$ in gage diameter, $25 \mathrm{~mm}$ in gage length, and $12 \mathrm{~mm}$ in diameter of grip section. For all experiments in this investigation the die temperature was kept at $220{ }^{\circ} \mathrm{C}$. For the purpose of comparison of tensile properties, the base alloy (LM24) was also sheared in the MCAST machine using similar superheat temperatures and processing conditions as described previously, and shaped to produce standard tensile test samples by HPDC.

Table I. Chemical Composition (Weight Percent) of the Al Alloy Used in This Study

\begin{tabular}{ccccccccccc}
\hline $\mathrm{Cu}$ & $\mathrm{Mg}$ & $\mathrm{Si}$ & $\mathrm{Fe}$ & $\mathrm{Mn}$ & $\mathrm{Ni}$ & $\mathrm{Zn}$ & $\mathrm{Pb}$ & $\mathrm{Sn}$ & $\mathrm{Ti}$ & $\mathrm{Al}$ \\
\hline 3.37 & 0.13 & 8.54 & 1.20 & 0.19 & 0.04 & 1.36 & 0.07 & 0.03 & 0.04 & $\mathrm{rem}$ \\
\hline
\end{tabular}


Table II. Process Variables Used with the MC-HPDC Method

\begin{tabular}{lll} 
MC-HPDC Processing Parameters & $\begin{array}{c}\text { Range of Processing } \\
\text { Parameters }\end{array}$ & \multicolumn{1}{c}{ Reason } \\
\hline Shearing time (s) & 60 to 240 & to optimize the cycle time \\
Shearing temperature $\left({ }^{\circ} \mathrm{C}\right)$ & 600 to 620 & to maintain optimum fluidity for casting \\
Shearing speed (rpm) & 400 to 800 & to apply sufficient hydrodynamic force \\
\hline
\end{tabular}

\section{Microstructures and Tensile Tests}

To characterize the uniformity of reinforcement, specimens were cut from different sections of the test samples. The specimens for microscopical observations were prepared by the standard technique of grinding with $\mathrm{SiC}$ abrasive papers, polishing with a diamond suspension solution. The microstructures were examined using an optical microscope (Carl Zeiss Axioskop 2 MAT0) and Zeiss Supra 35VP FEG scanning electron microscope (SEM). The tensile tests properties were measured using a universal materials testing machine (INSTRON* 5569) at a crosshead speed of

*INSTRON is a trademark of Instron Corporation, Canton, MA.

$2 \mathrm{~mm} /$ minute (strain rate: $1.33 \times 10^{-3} \mathrm{~s}^{-1}$ ).

The homogeneity of a distribution forms a complex problem that has been addressed in metallography by means of a broad spectrum of techniques. ${ }^{[48-53]}$ One of the simplest and most effective in detecting pronounced changes in the MMC microstructure is the quadrat method. ${ }^{[53]}$ With the quadrat method the image to be studied is divided into grid of square cells where the square quadrat size is approximately twice the size of the mean area per particle. ${ }^{[54]}$ The quadrat method was performed on 50 images of each specimen within square fields, $924 \times 924$ pixels in size. Each field was divided into 36 contiguous quadrats. A quadrat size of $154 \times 154$ pixels $(20 \times 20 \mu \mathrm{m})$ was chosen at 500 times

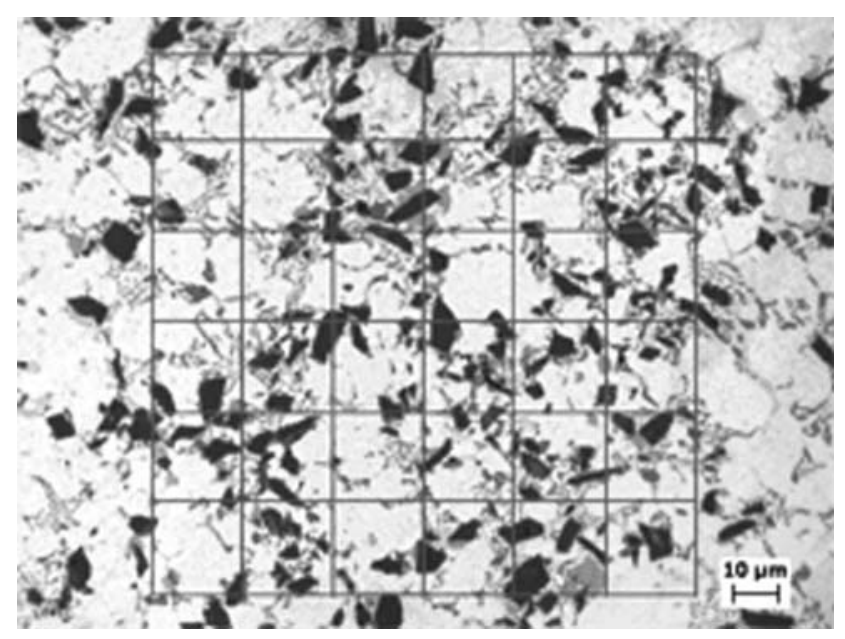

Fig. 4-Representative optical micrograph from LM24-10 vol pct $\mathrm{SiC}_{p}$ composite for analysis by the quadrat method. magnification (Figure 4). Problems associated with image analysis could result in errors such as (a) pronounced clustering and touching of $\mathrm{SiC}$ particles in a cast MMC leads to identify some of them as one particle as it is based on gray color differences among the features; and (b) distinguishing very small $\mathrm{SiC}$ particles from artifacts, such as intermetallic particles and pores. These problems can be minimized by carefully defining the size range for measurement of the particles and increasing the magnification. The degree of asymmetry of a statistical distribution around its mean can be quantified by its skewness $\beta$, which is defined by

$$
\beta=\frac{q}{(q-1)(q-2)} \sum\left[\frac{N_{q i}-N_{q}^{\text {mean }}}{\sigma}\right]^{3}
$$

where $q$ is the total number of quadrats studied, $N_{q i}$ is the number of $\mathrm{SiC}$ particles in the $i$ th quadrat $(i=1$, $2, \ldots \ldots, \mathrm{q}), N_{q}^{\text {mean }}$ is the mean number of $\mathrm{SiC}$ particles per quadrat, and $\sigma$ is the standard deviation of the $N_{q}$ distribution. According to a previous study, ${ }^{[53]}$ an increase in $\beta$ indicates an increase in $\mathrm{SiC}$ clustering.

\section{RESULTS AND DISCUSSION}

\section{A. General Microstructure}

Optical microscopy carried out on the conventional HPDC samples containing 5 and 10 pct volume percentage of $\mathrm{SiC}$ revealed the presence of sites of $\mathrm{SiC}$ particulates clustering and volumes free of $\mathrm{SiC}$ particulates, whereas MC-HPDC samples show an improvement in the dispersion of $\mathrm{SiC}$ particulates in all cases (Figure 5). During the conventional stirring process, axial flow causes lifting of particles due to momentum transfer and radial flow prevents particle settling. A lack of sufficient hydrodynamic forces results in accumulation of aggregates in relatively stagnant zones (e.g., near the crucible walls) where they survive the shear forces of mixing and are not transported back into the high shear regions to find their way as clusters into the cast structure. The fluid flow inside the slurry maker is characterized by a cyclic variation of a high shear rate, high intensity of turbulence, and positive displacement pumping action. The high shear regions are schematically shown in Figure 6.

During dispersive mixing process, there is an enormous amount of ever-changing interfacial area between the composite mixture and the slurry maker. The lowest shear rate is found at the gap between the root of the 

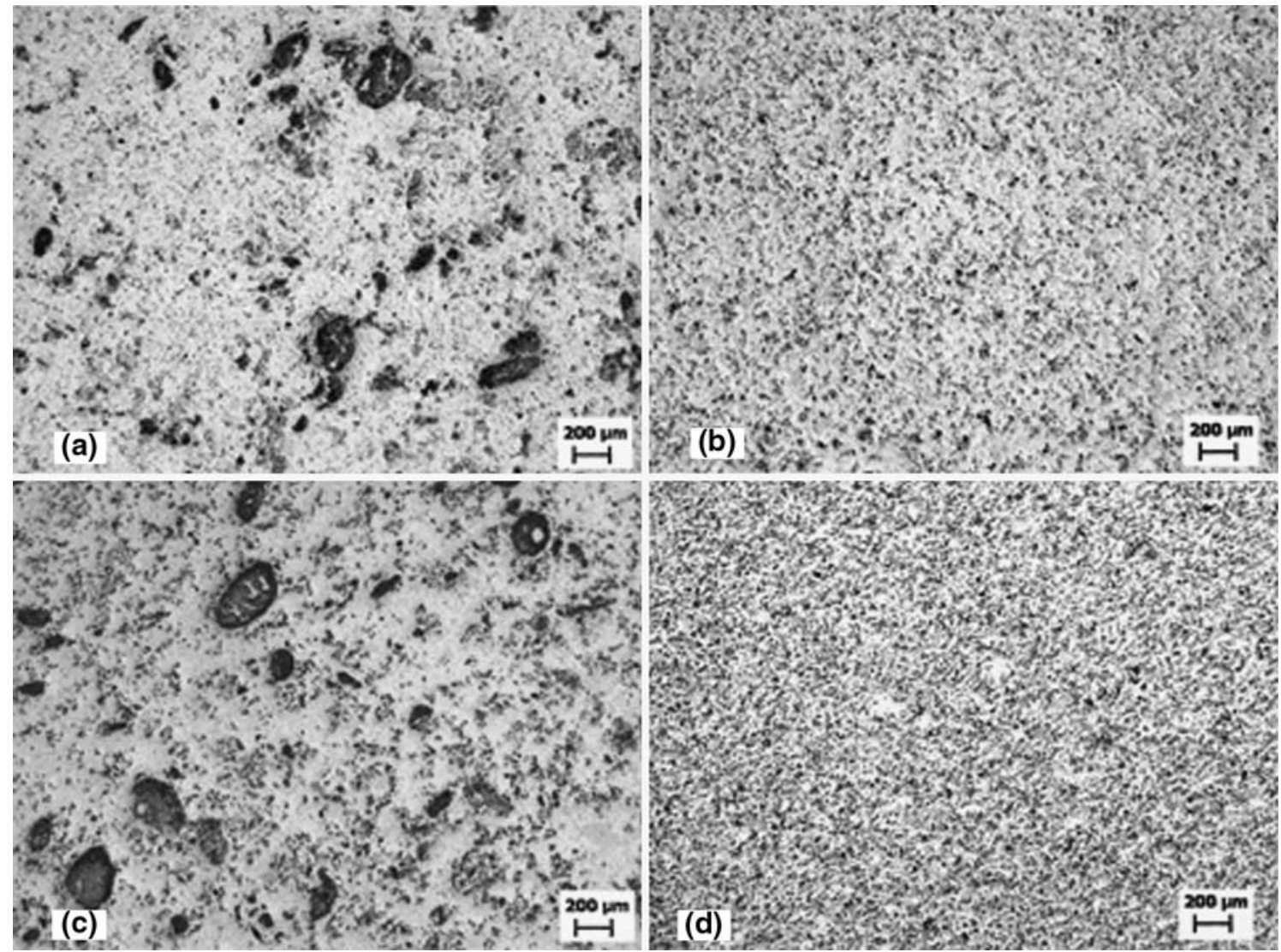

Fig. 5- (a) Representative optical micrograph taken from LM24-5 vol pct SiC composite sample in the conventional HPDC. (b) Representative optical micrograph taken from LM24-5 vol pct SiC composite sample in the MC-HPDC. (c) Representative optical micrograph taken from LM24-10 vol pct SiC composite sample in the conventional HPDC. (d) Representative optical micrograph taken from LM24-10 vol pct SiC composite sample in the MC-HPDC.

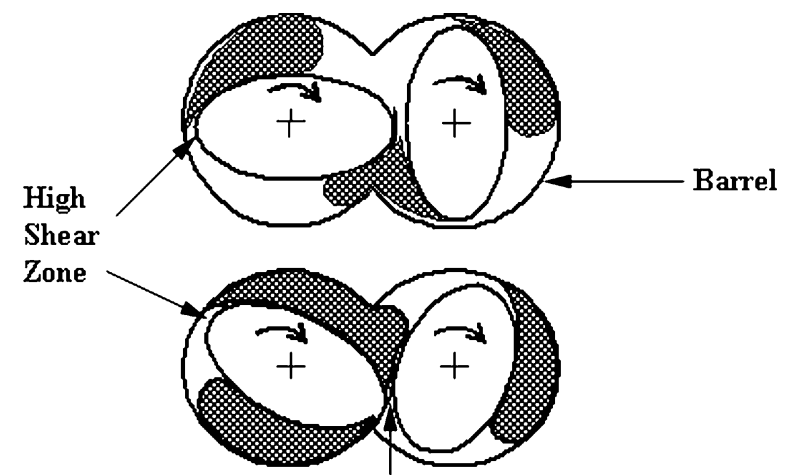

High Shear Zone

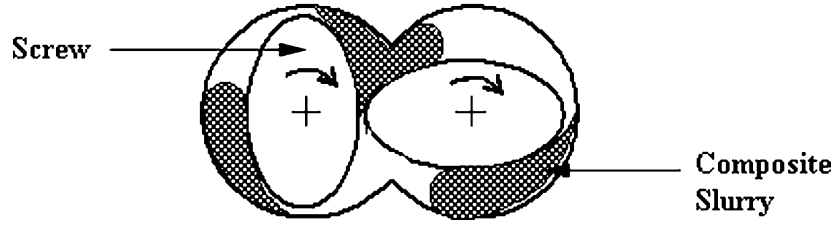

Fig. 6-Schematic illustration of fluid flow and high shear zones during dispersive mixing with twin screw. ${ }^{[55]}$

screw flight and the inner surface of the barrel; the highest shear rate is offered by the intermeshing regions between the two screws. Based on the fluid flow, the shear stress between the screw and the barrel in a MCAST machine can is given by ${ }^{[56]}$

$$
\tau=\eta \pi N\left(\frac{D}{G}-2\right)
$$

where $\eta$ is the viscosity, $N$ is the rotation speed of the screw, $D$ is the outer diameter of the screw, and $G$ is the gap between the screw flight and the barrel surface. Hydrodynamic stresses developed due to the specially designed profile of the screws break the agglomerates into individual particles and result in a homogeneous dispersion. Equation [4] should be invoked during a discussion of the results of the processing variables such as shearing time, shearing speed, and apparent viscosity on the quality of the distribution obtained in cast samples. The analysis and supporting micrographs from previous studies $^{[21-24]}$ clearly showed that the reinforcement distribution in the cross sections of the cast parts was not uniform. Particulate clustering or some segregation bands were observed in the microstructure of different sections of the die-cast part. However, MCHPDC samples reveal a uniformity of the reinforcement distribution as shown in Figure 7 . The image analysis on microstructures of $\mathrm{LM} 24-5$ pct $\mathrm{SiC}_{p}$ and $\mathrm{LM} 24-10$ pet $\mathrm{SiC}_{p}$ has shown the reinforcement volume percentages to be $5.26 \pm 0.91$ and $10.35 \pm 1.12$ pct, respectively. 


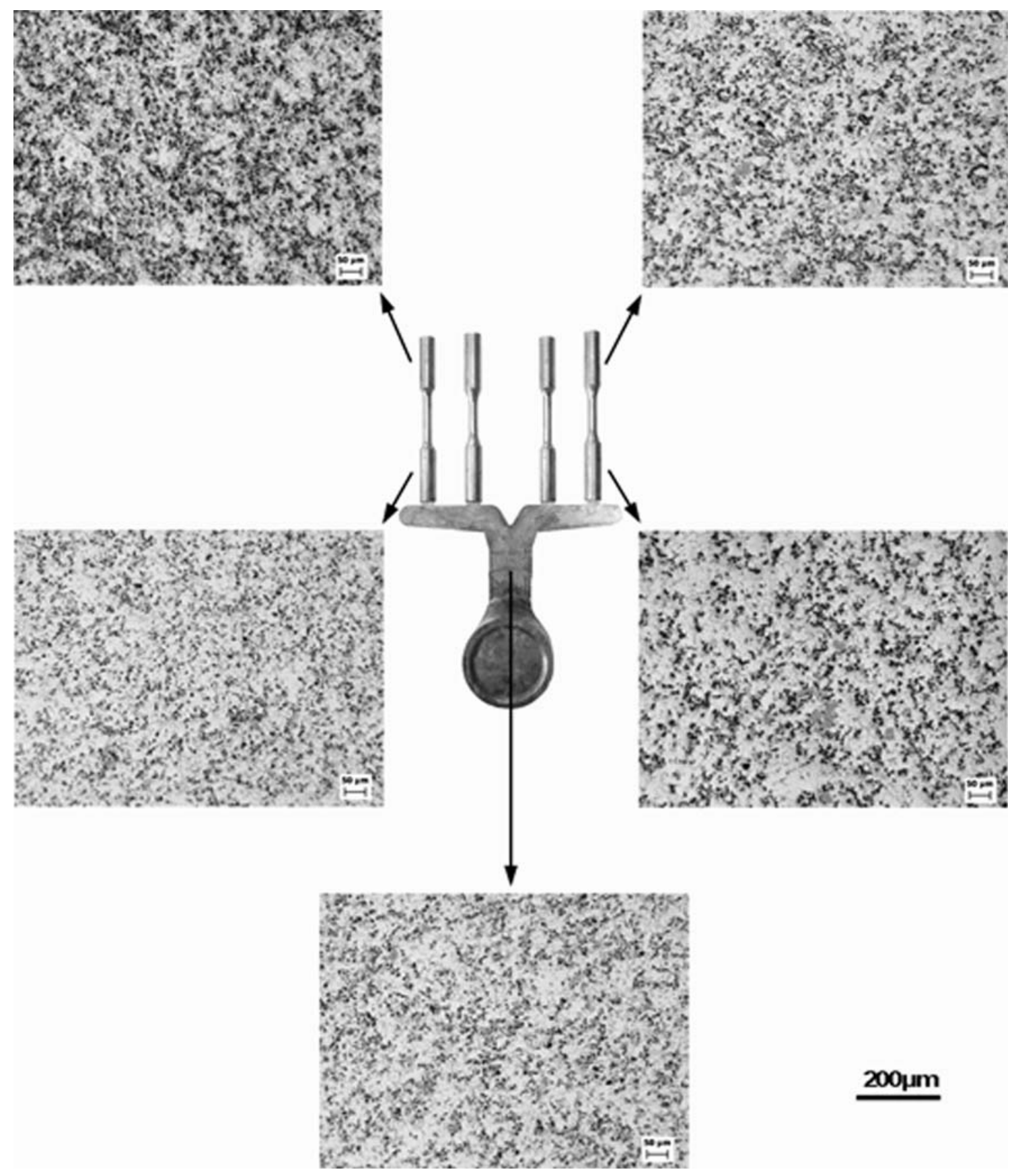

Fig. 7- Optical micrographs taken from different locations of a cast specimen produced by MC-HPDC process having a 10 vol pct SiC content.

B. Effect of Shearing Time and Temperature on Degree of Homogeneity

Experiments were carried out to investigate the effects of shearing time on the distribution of ultrafine-sized $\mathrm{SiC}$ particles in the matrix. The calculated skewness $(\beta)$, an indicative of degree of asymmetry, is plotted in Figure 8 as a function of shearing time for LM24-5 vol pct SiC composites.

Figure 8 indicates that dispersive mixing in the MCAST machine has a significant positive effect on the distribution of $\mathrm{SiC}$ particles compared to the conventional mixing process. With MC-HPDC composite material, $\beta$ ranges from 0.76 for a sample processed at $600{ }^{\circ} \mathrm{C}$ and 180 seconds, to 1.39 for a sample processed at $610{ }^{\circ} \mathrm{C}$ and 60 seconds. The corresponding value is 2.37 for the conventional HPDC composite material. When the shearing time was increased from 60 to 180 seconds, the skewness $\beta$ decreased and then increased at 240 seconds. An increased shearing time dissociates the interaggregate bonds but can also increase the frequency of collision between these particles resulting in the formation of new agglomerates, which impair the mixing quality. ${ }^{[13]}$ From Eq. [4] it is clear that the shear stress is independent of shearing time. 


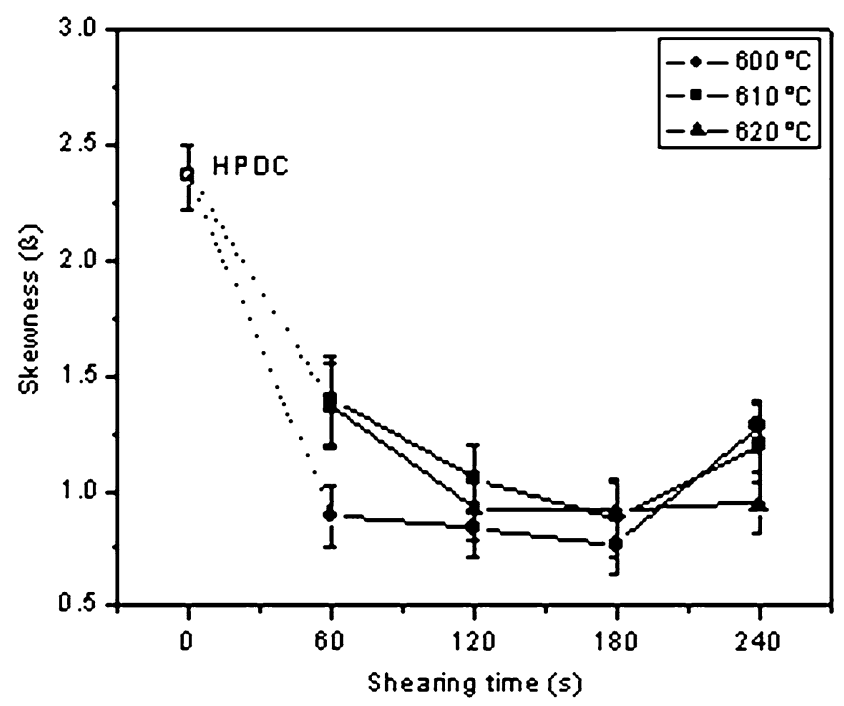

Fig. 8-Skewness as a function of shearing time at rotation speed of $800 \mathrm{rpm}$ for MC-HPDC samples.

Therefore, a significant variation was not observed for the MC-HPDC composite samples. A shorter cycle time improves the performance in terms of production cost and interfacial bond strength between reinforcements and the matrix.

Figure 8 also indicates that decreasing the processing temperature from $620^{\circ} \mathrm{C}$ to $600{ }^{\circ} \mathrm{C}$ enhances mixing quality. The apparent viscosity increases as temperature decreases, which in turn increases the shear stress $(\tau)$. An increased shear stress breaks up the agglomerates and disperses particles uniformly. An increase in total surface area of ultrafine-size particulates causes more resistance to fluid flow as a result of stagnant boundary layers around the particles. ${ }^{[57]}$ Therefore, to improve the fluidity of the $\mathrm{Al} / \mathrm{SiC}$ slurry and to die cast the sheared slurry into the required shapes, it is necessary to work above liquidus temperature of alloy. The HPDC experiments are carried out above liquidus temperature (approximately $630{ }^{\circ} \mathrm{C}$ ) and hence connected by a dotted line.

\section{Effect of Shearing Intensity on Degree of Homogeneity}

Figure 9 presents the variation in skewness as a function of shearing speed. When the screw speed rotation is increased from 400 to $800 \mathrm{rpm}$, the skewness during the shearing process decreased by 69 pct for LM24-10 pet SiC composite and 15.32 pct for the LM24-5 pct SiC. The decrease in skewness with an increase in shearing speed is because of the increase in shear stress with rotational speed. The viscosity increases with the volume fraction of the reinforcement and with a decrease in the particle size. ${ }^{[57,58]}$ During shearing at a constant shear stress, increasing the volume fraction of reinforcing particles increases the interaction between them. The applied shear stress is proportional to the rotational speed and viscosity of the slurry. An increase in shear stress breaks up the

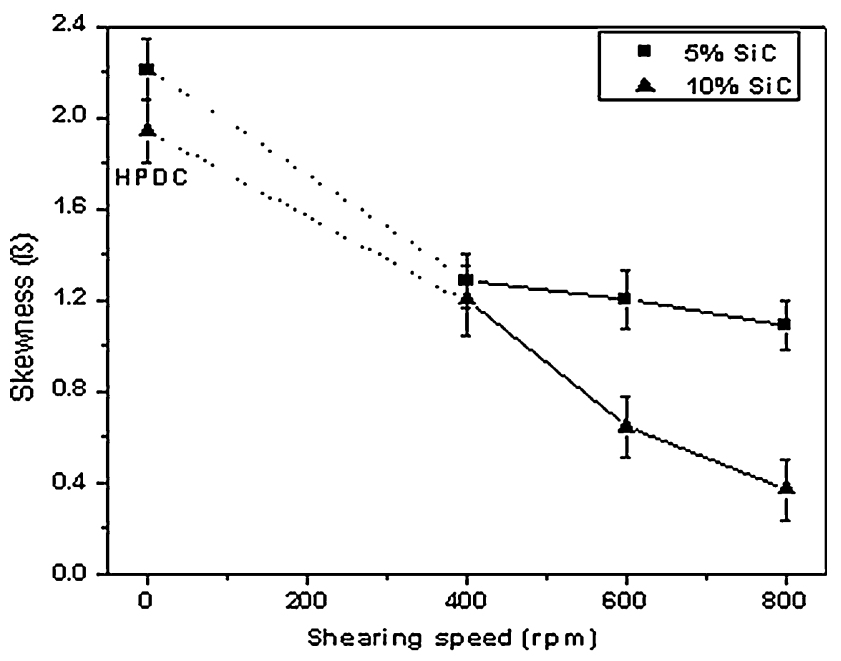

Fig. 9- Skewness as a function of shearing speed at $610^{\circ} \mathrm{C}$ and $120 \mathrm{~s}$.

agglomerates by overcoming the cohesive forces that hold them together. Under a high shear rate and turbulent conditions the melt penetrates into the clusters and disperses the individual particles within the cluster, resulting in a more uniform distribution. The shear stress $(\tau)$ predicted by Eq. [4] also suggests that an increase in rotational speed of the screws and viscosity increases the applied shear stress and contributes to inhomogeneity of the reinforcement distribution.

\section{Quantitative Analysis}

The quadrat method is evidently an effective method of detecting variations in the distribution of $\mathrm{SiC}$ particulates in MMCs induced by processing. In mathematical terms, theoretically random, spatial, and clustered spatial distributions of particles can be expressed by a Poisson distribution, a binomial distribution, and a negative binomial distribution, respectively. ${ }^{[59]}$ The experimental results from the quadrat analysis can be compared with theoretical distribution curves in absolute terms. ${ }^{[53]}$

Number of particles in a quadrat $\left(N_{q}\right)$ was measured. Number of quadrats containing $N_{q}$ particles $\left(N_{q}=0,1\right.$, $2,3 \ldots, 12)$ are presented in terms of frequency (Figure 10). The observed distribution for composite synthesized by the conventional HPDC process follows a clustered distribution expressed by a negative binomial curve, whereas the corresponding distribution for the MC-HPDC composite shows a pronounced deviation from the negative binomial distribution and is closer to both the Poisson and the binomial distributions, indicating a more uniform distribution (Figure 10).

\section{E. Interfacial Analysis}

The interface formed between the matrix and the ceramic reinforcement determines the load transfer and crack resistance of the MMCs during deformation. The $\mathrm{SiC}$ particles are known to react with liquid aluminum to form a layer of reaction product $\left(\mathrm{Al}_{4} \mathrm{C}_{3}\right)$ at the 


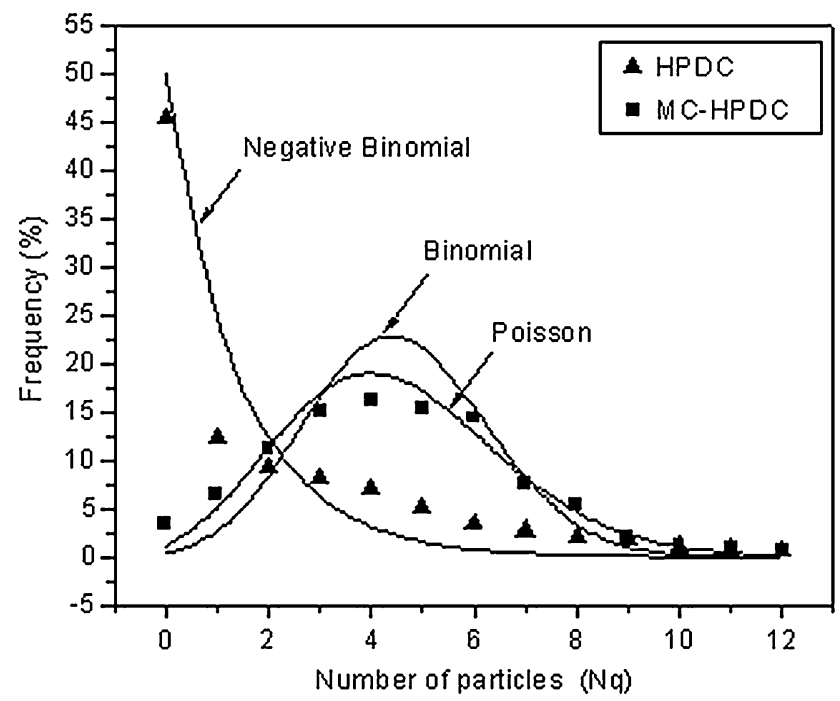

Fig. 10-Theoretical distribution curves and experimental results (symbols) from a quadrat analysis for LM24-10 vol pet $\mathrm{SiC}_{p}$.

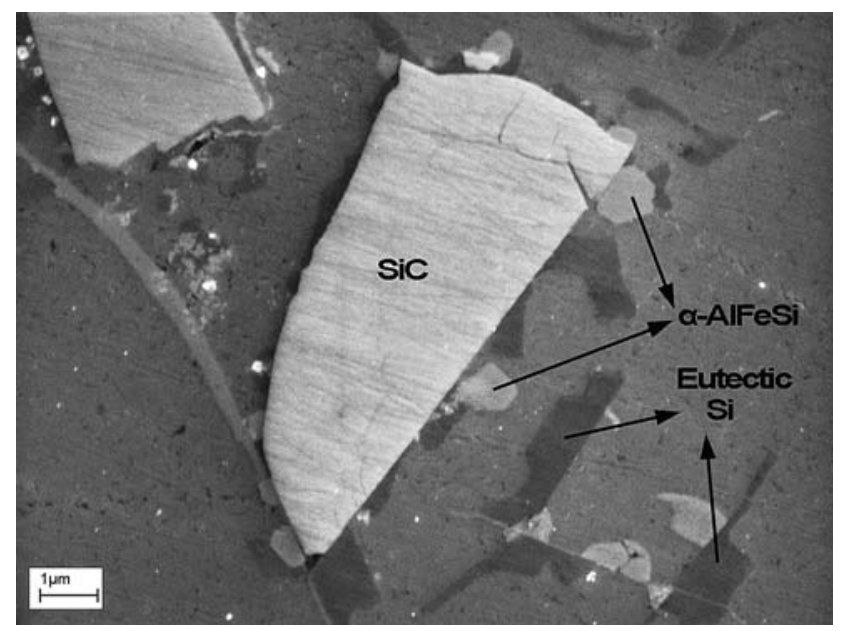

Fig. 11-SEM image showing the $\mathrm{SiC} / \mathrm{Al}$ interface.

reinforcement-matrix interface, which degrades the mechanical properties of the composites, particularly at elevated temperatures. ${ }^{[2,4]}$ The LM24-10 vol pet of $\mathrm{SiC}_{p}$ specimen processed at $610{ }^{\circ} \mathrm{C}, 600 \mathrm{rpm}$, and sheared for 120 seconds, was polished and cleaned ultrasonically in ethanol for SEM observations.

In Figure 11, the interface between $\mathrm{SiC}$ and the $\mathrm{Al}$ matrix was observed to be sharp and clean, indicating that no interfacial chemical reaction had occurred such as observed by Tham et al. ${ }^{[1]}$ This is attributed to the short cycle time of the MC-HPDC process and the high Si content $(8.54 \mathrm{wt} \mathrm{pct})$, which avoids the interfacial reaction under the processing contact time and temperature. $^{[45]}$

\section{F. Tensile Properties of LM24-SiC Composite $^{2}$}

It is well known that both the structure and properties of cast composites are extremely sensitive to the

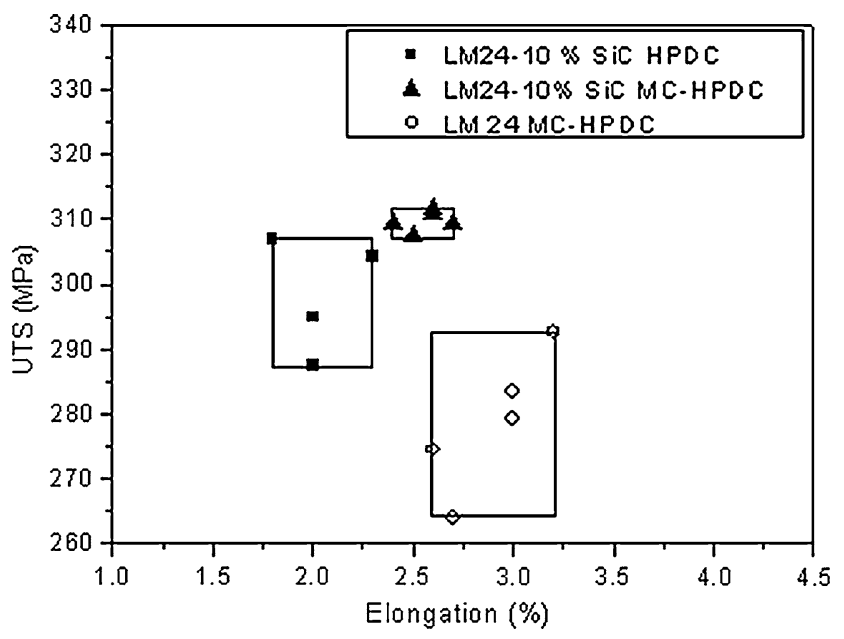

Fig. 12-Comparison of tensile properties of LM24 and LM24-10 vol pct $\mathrm{SiC}_{p}$ samples obtained from different processing techniques.

fabrication techniques. A large amount of mechanical property data for various composites is available in Reference 6. Compared with the conventional HPDC process, the MC-HPDC process provides samples with a slightly higher ultimate tensile strength and much higher tensile elongation. The elongation of the MC-HPDC samples is approximately 20 pct higher than that of HPDC samples. This is a very important feature of the MC-HPDC process. The MC-HPDC samples had negligible porosity. Therefore, the mechanical properties can be further enhanced through heat treatment.

Figure 12 shows a comparison of properties from tensile tests for LM24-10 pct $\mathrm{SiC}$ composite samples processed by the two techniques. Figure 12 clearly demonstrates that the MC-HPDC process offers better tensile properties than the conventional HPDC process in terms of increased strength and ductility simultaneously. The uniform distribution of reinforcement within the matrix achieved by the MC-HPDC process directly impacts on the properties and quality of the composite material. The observed property data for melt-conditioned base alloy (LM24) is also reported in Figure 12. The results of the present study revealed a superior strength and reasonably comparable ductility of the MC-HPDC composite samples when compared to the unreinforced samples.

A ceramic reinforcement incorporated in a metal matrix increases the effective composite modulus. For a twocomponent composite, the Hashin-Shtrikman bounds ${ }^{[60]}$ would give a good estimate of expected modulus. The Hashin-Shtrikman bounds are valid for all isotropic random materials and information required to calculate these bounds are volume percentage and elastic properties of each phase. The Hashin-Shtrikman bounds of an expected elastic modulus $\left(\varepsilon_{e}\right)$ are calculated by

$$
\varepsilon_{m}+\frac{\varepsilon_{m} V_{f}}{\frac{\varepsilon_{m}}{\left(\varepsilon_{f}-\varepsilon_{m}\right)}+\frac{V_{m}}{3}}<\varepsilon_{e}<\varepsilon_{f}+\frac{\varepsilon_{f} V_{m}}{\frac{\varepsilon_{f}}{\left(\varepsilon_{m}-\varepsilon_{f}\right)}+\frac{V_{f}}{3}}
$$

where $V_{m}$ is the volume fraction of the matrix phase, $V_{f}$ is the volume fraction of the reinforcement phase, and 


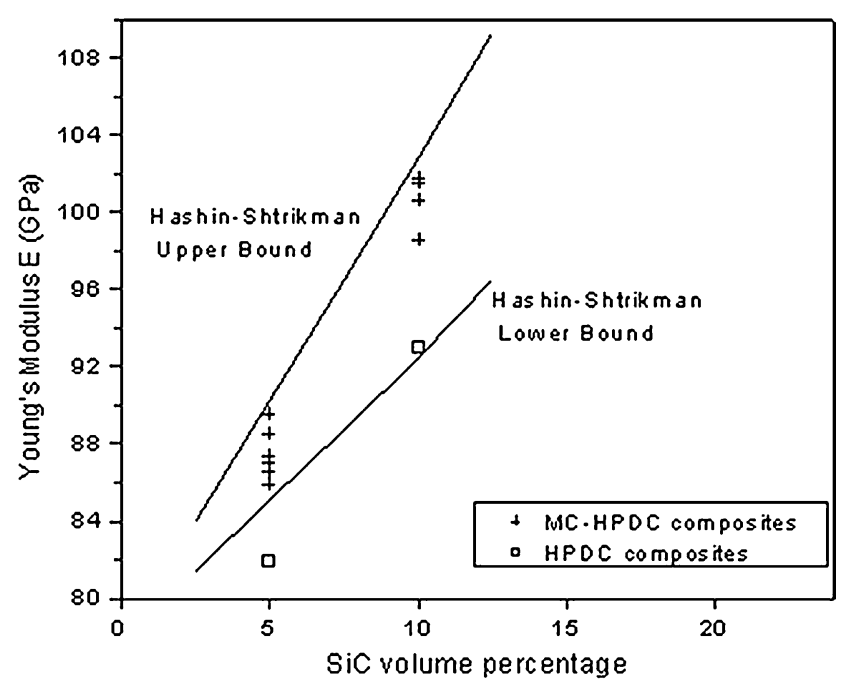

Fig. 13-Hashin-Shtrikman bounds and observed values of Young's modulus for composites.

$\varepsilon_{m}$ and $\varepsilon_{f}$ are modulus of matrix and reinforcement phase respectively. The observed value of Young's modulus for the melt-conditioned LM24 alloy is $77.96 \pm 2 \mathrm{GPa}$. The Young's modulus value provided by the supplier for the $\mathrm{SiC}_{p}$ is $410 \mathrm{GPa}$. Based on these data, the Hashin-Shtrikman bounds (solid lines in Figure 13) of Young's modulus for 5 and $10 \mathrm{vol}$ pet and corresponding observed values (data points in Figure 13) during tensile tests are plotted.

Figure 13 shows that Young's modulus values of MCHPDC composites fall within the Hashin-Shtrikman bounds. In accordance with previous studies ${ }^{[6]}$ the elastic modulus of discontinuously reinforced composites increases with increasing additions of the ceramic reinforcement. The major source of divergence of Young's modulus value for LM24-5 pet SiC HPDC composite from the theoretical value is the presence of clusters and agglomerates as seen in Figure 5(a). The small variation observed in Young's modulus of MC-HPDC composites synthesized with different processing conditions is due to the fact that, compared to the mechanical strength, the composite modulus is relatively insensitive to the reinforcement distribution ${ }^{[6]}$ However, better tensile properties of MC-HPDC composites also indicate improved interfacial bond strength between the reinforcement and the matrix.

\section{CONCLUSIONS}

A rheo-processing technology, MC-HPDC has been developed for the production of high-performance Al-SiC composite material. The ultrafine $\mathrm{SiC}$ particulates (average size as small as $4 \mu \mathrm{m}$ ) were successfully introduced into the $\mathrm{Al}$ matrix using a liquid processing route. Intensive shearing helped to break up the agglomerates and disperse the particles uniformly throughout the melt. It is observed that prolonged shearing impairs the mixed quality and increased shear intensity enhances the distribution of reinforcing particles. Quantitative analysis revealed that MC-HPDC offers components of high integrity compared with the conventional HPDC process. Much improved tensile properties, particularly elongation, are achieved due to structural uniformity and the absence of interfacial reaction product. A shorter cycle time and high shear dispersive mixing improve the performance in terms of production cost and efficiency.

\section{ACKNOWLEDGMENTS}

The authors acknowledge contribution to this work from Dr. J. Patel and the financial support from EPSRC.

\section{REFERENCES}

1. P. Rohatgi: AFS Trans., 2001, pp. 1-133.

2. I.A. Ibrahim, F.A. Mohamed, and E.J. Lavernia: J. Mater. Sci., 1991, vol. 26, pp. 1137-56.

3. D.J. Lloyd: Int. Mater. Rev., 1994, vol. 39 (1), pp. 1-23.

4. S. Ray: J. Mater. Sci., 1993, vol. 28, pp. 5397-5413.

5. D.B. Miracle: Compos. Sci. Technol., 2005, vol. 65, pp. 2526-40.

6. R. Asthana: Adv. Perf. Mater., 1998, vol. 5, pp. 213-55.

7. N. Chawala and K.K. Chawala: J. Mater., 2006, vol. 58 (11), pp. 67-70.

8. H. Rumpf: Agglomeration, Interscience Publishers, New York, NY, 1962, pp. 379-413.

9. S.V. Nair, J.K. Tien, and R.C. Bates: Int. Met. Rev., 1985, vol. 30 (6), pp. 275-90.

10. D.J. Lloyd: Acta Metall. Mater., 1991, vol. 39 (1), pp. 59-71.

11. L.M. Tham, M. Gupta, and L. Cheng: Acta Mater., 2001, vol. 49, pp. 3243-53.

12. F. Rana, B.K. Dhindaw, and D.M. Stefanescu: AFS Trans., 1989, pp. 255-64.

13. P.K. Rohatgi, J. Sobczak, R. Asthana, and J.K. Kim: Mater. Sci. Eng. A, 1998, vol. A252, pp. 98-108.

14. N. Aniban, R.M. Pillai, and B.C. Pai: Mater. Des., 2002, vol. 23, pp. 553-56.

15. K.R. Ravi, V.M. Sreekumar, R.M. Pillai, C. Mahato, K.R. Amaranathan, R.A. Kumar, and B.C. Pai: Mater. Des., 2007, vol. 28, pp. 871-81.

16. P.K. Rohatgi, R. Asthana, and S. Das: Int. Met. Rev., 1986, vol. 31 (3), pp. 115-39.

17. A. Mortensen and I. Jin: Int. Mater. Rev., 1992, vol. 37 (3), pp. 101-28.

18. J. Hashim, L. Looney, and M.S.J. Hashmi: J. Mater. Process. Technol., 2002, vol. 123, pp. 251-57.

19. J. Hashim, L. Looney, and M.S.J. Hashmi: J. Mater. Process. Technol., 2002, vol. 123, pp. 258-63.

20. D.M. Stefanescu, B.K. Dhindaw, S.A. Kacar, and A. Moitra: Metall. Trans. A, 1988, vol. 19A, pp. 2847-855.

21. C.B. Lin, C.L. Ma, and Y.W. Chung: J. Mater. Process. Technol., 1998, vol. 48, pp. 236-46.

22. C.B. Lin, C.L. Wu, and C.H. Chiang: J. Mater. Sci., 1999, vol. 34, pp. $2229-40$.

23. H. Sevik and S. Can Kurnaz: Mater. Des., 2006, vol. 27, pp. 67683.

24. Z. Zhang, X.G. Chen, and A. Charette: J. Mater. Sci., 2007, vol. 42, pp. 7354-62.

25. F. Delannay, L. Froyen, and A. Deruyttere: J. Mater. Sci., 1987, vol. 22, pp. 1-16.

26. M.K. Surappa and P.K. Rohatgi: J. Mater. Sci., 1981, vol. 16, pp. 983-93.

27. M. Gupta and M.K. Surappa: Key Eng. Mater., 1995, vols. 104 107, pp. 259-74. 
28. W.C. Harrigan, Jr.: Mater. Sci. Eng. A, 1998, vol. 244A, pp. 75-79.

29. T.S. Srivatsan, M. Al-Hajri, C. Smith, and M. Petraroli: Mater. Sci. Eng., 2003, vol. 346A, pp. 91-100.

30. A. Onat, H. Akbulut, and F. Yilmaz: J. Alloy Compd., 2007, vol. 436 , pp. $375-82$

31. S. Skolianos: Mater. Sci. Eng. A, 1996, vol. 210A, pp. 76-82.

32. M. Gupta, L. Lu, M.O. Lai, and S.E. Ang: Mater. Des., 1995, vol. 16 (2), pp. $75-81$.

33. C.G. Kang and S.W. Youn: J. Mater. Process. Technol., 2004, vol. 147, pp. 10-22.

34. Y. Yalcin and H. Akbulut: Mater. Des., 2006, vol. 27, pp. 872-81.

35. J. Segurado, C. Conzalez, and J. Llorca: Acta Mater., 2003, vol. 51, pp. 2355-69.

36. X. Deng and N. Chawla: J. Mater. Sci., 2006, vol. 41, pp. 5731-34.

37. J. Tomas: Chem. Eng. Sci., 2007, vol. 62, pp. 1997-2010.

38. M.L. Falk and J.S. Langer: Phys. Rev. E, 1998, vol. 57 (6), pp. 7192-205.

39. C.A. Schuh and A.C. Lund: Nat. Mater., 2003, vol. 2, pp. 449-52.

40. N. Harnby, M.F. Edward, and A.W. Nienow: Mixing in the Process Industries, 2nd ed., Butterworths-Heinemann Ltd., Oxford, United Kingdom, 1985, pp. 99-136.

41. W. Beck, P. Forschner, R. Junghanns, D. Klatt, G. Philipp, F. Sauter, H. Schneider, T. Schneider, K.-H. Schwarzer, W. Schwechheimer, R. Vettermann, and E. Zimmermann: Handbook of Mixing Technology, EKATO Ruhr-und Mischtechnik GmbH, Schopfheim, Germany, 1991, pp. RS1-RS10.

42. K. Kendall: Powder Metall., 1988, vol. 31 (1), pp. 28-31.

43. Z. Fan, M.J. Bevis, and S. Ji: UK Patent Application No. 9922696.3, 1999.

44. S. Ji, Z. Fan, and M.J. Bevis: Mater. Sci. Eng. A, 2001, vol. 299A, pp. 210-17.
45. J.C. Lee, J.Y. Byun, S.B. Park, and H.I. Lee: Acta Mater., 1998, vol. 46 (5), pp. 1771-80.

46. Z. Fan, X. Fang, and S. Ji: Mater. Sci. Eng. A, 2005, vol. 412A, pp. 298-306.

47. H. Tang, L.C. Wrobel, and Z. Fan: Modell. Simul. Mater. Sci. Eng., 2003, vol. 11, pp. 771-90.

48. S. Yotte, D. Breysse, J. Riss, and S. Ghosh: Mater. Charact., 2001, vol. 46, pp. 211-19.

49. P. Ganguly and W.J. Poole: Mater. Sci. Eng. A, 2002, vol. 332A, pp. $301-10$.

50. I.G. Waston, M.F. Forster, P.D. Lee, R.J. Dashwood, R.W. Hamilton, and A. Chirazi: Composites Part A, 2005, vol. 36, pp. 1177-87.

51. J.E. Spowart: Mater. Sci. Eng. A, 2006, vol. 425A, pp. 225-37.

52. J.E. Spowart, B. Maruyama, and D.B. Miracle: Mater. Sci. Eng. A, 2001, vol. 307A, pp. 51-66.

53. P.A. Karnezis, G. Durrant, and B. Cantor: Mater. Charact., 1998, vol. 40, pp. 97-109.

54. J.T. Curtis and R.P. McIntosh: Ecology, 1950, vol. 31, pp. 434-55.

55. J.L. White: Twin Screw Extrusion: Technology and Principles, Hanser Publisher, Munich, Germany, 1990, p. 229.

56. C. Rauwendaal: Polymer Extrusion, 3rd rev. ed., Hanser Publisher, New York, NY, 1994, p. 181.

57. K.R. Ravi, R.M. Pillai, K.R. Amaranathen, B.C. Pai, and M. Chakraborty: J. Alloy Compd., 2008, vol. 456 (1-2), pp. 201-10.

58. M.C. Fleming: Metall. Mater. Trans. A, 1991, vol. 22 (5), pp. 975 81.

59. A. Rogers: Statistical Analysis of Spatial Dispersion: The Quadrat Method, 3rd ed., Pion, London, United Kingdom, 1974, pp. 1-164.

60. T.C. Choy: Effective Medium Theory: Principles and Applications, 1st ed., Oxford University Press, Oxford, United Kingdom, 1999, pp. 24-30. 